\title{
ANALYSIS AND SIMULATION OF SEISMIC GROUND MOTIONS OBSERVED BY AN ARRAY IN A SEDIMENTARY BASIN
}

\author{
Masanori HORIKE \\ Department of Architectural Engineering, Osaka Institute of \\ Technology, Osaka, Japan
}

(Received February 3, 1988; Revised September 1, 1988)

\begin{abstract}
The effects of the irregularity of a shallow subsurface interface on seismic motions are investigated using aftershock records of the western Nagano Prefecture earthquake of September 14, 1984, observed by an array in the Kyoto basin extending from the rock outcrop near the eastern margin to the center. Comparison between low-pass-filtered seismograms at the basin sites indicates the presence of spatial waveform variation and elongation of duration of the S-wave portion (from the onset of $S$ wave to a later part where amplitudes decrease to a certain low level). The phase and group velocities of the wavelets contained in the S-wave portion suggest that the above characteristics of seismic motion in the Kyoto basin are primarily caused by the excitation of surface waves at the eastern margin of the basin. This means that the prediction of seismic motions in a basin-like structure requires the consideration of irregular interfaces. To reproduce the recorded seismograms in the Kyoto basin, the Aki and Larner method is extended to allow the computation of seismic responses of a two-dimensional basin-like structure with multiple irregular interfaces. The computed shear waves exhibit spatial waveform variation and elongation of duration due to surface waves generated at the eastern margin of the basin. However, the amplitude of the computed surface waves are less than the observed amplitude. To obtain better agreement, the other effects such as those of three-dimensional subsurface structure and non-plane wave front of incident motions should be included in the synthetic seismograms.
\end{abstract}

\section{Introduction}

Estimation of the intensity and duration of seismic motions in sedimentary basins and plains, which often contain large population densities, is one of the most urgent problems for earthquake-hazard mitigation. In order to estimate them, we have to evaluate not only the effects of seismic source and propagation path but also those of local geological conditions such as soft sediments, basin-bedrock interface, and surface topography on seismic motions.

A prominent feature of subsurface structure in sedimentary basins is an irregular basin-bedrock interface with high contrast in velocity and density. It may generate characteristic wave phenomena such as surface waves and resonance that 
may result in the spatial variation in intensity and duration. In recent years, many investigators have shown that the irregularity of the basin-bedrock interface strongly influences seismic motions in a sedimentary basin. ToRIUmi (1980) first found that surface waves were secondarily generated at the margin of the Osaka plain. Since then, the same phenomenon has been observed by other investigators (for example, Kudo, 1980; LiU and Heaton, 1984; Kinoshita, 1986; Sasatani, 1985; Minamishima et al., 1986). On the other hand, King and TUCKer (1984) found resonance in sediment-filled valleys in the Garm region of the USSR.

Meanwhile, various numerical simulation methods also revealed the strong influence of the irregularity of the basin-bedrock interface on seismic motions (for example, AKI and LARNER, 1970; TrIFUnAC, 1971; LySMER and DraKe, 1971; BoOre, 1972; Sanchez-Sesma and Esquivel, 1979; Dravinsky, 1983; ČERvenÝ et al., 1987). In particular, BARD and BOUCHON (1985) numerically demonstrated that the occurrence of the two phenomena (surface wave or resonance) was controlled primarily by the shape ratio of the basin (the ratio of the maximum depth to the half width) and the contrast between $\mathrm{S}$-wave velocities of the basin and the bedrock.

However, these observational and numerical studies were made separately so that the effects of the irregularity of interfaces on seismic motions have not been quantitatively investigated until now. To make such an investigation, we first of all have to choose a sedimentary basin where the subsurface structure is already known. The Kyoto basin shown in Fig. 1(a) is such a basin. Thus, we investigate the effects of irregular interfaces on seismic motions here in the Kyoto basin. The present study has two aims. The first one is to investigate what kind of wave phenomenon is associated with the irregularity of the basin-bedrock interface based on analyses of seismic array data observed in the Kyoto basin. The second is to evaluate quantitatively the effects of the irregularity of the interfaces on seismic motions. The latter is done by comparison between recorded seismograms in the Kyoto basin and synthetic seismograms calculated using the extended Aki and Larner method (hereafter abbreviated as the AL method). This method allows the computation of responses of a two-dimensional subsurface structure with multiple irregular interfaces.

\section{Observations and Data}

Seismic observations have been made at three sites in the Kyoto basin as shown in Fig. 1(b). One site (OBK) is on a rock outcrop at the eastern margin of the basin. Another site (KUC) is nearby on soft sediments. The other site (OGR) is slightly to the east of the center of the basin. At OGR, a tripartite subarray was established to distinguish wave types (surface waves or body waves). The subarray configuration is shown in Fig. 1(c). All the seismometers used in this study were velocity sensors with a natural period $2 \mathrm{~s}$. A clock was common at the two sites OBK and KUC, and another clock was used at the site OGR. However, the time difference between the two clocks did not exceed $0.3 \mathrm{~s}$. 
(a)

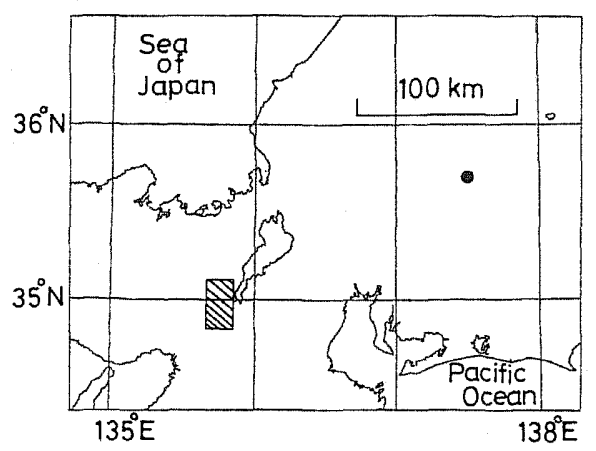

(c)

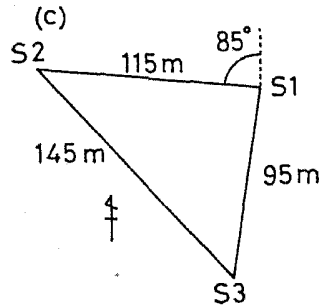

(b)

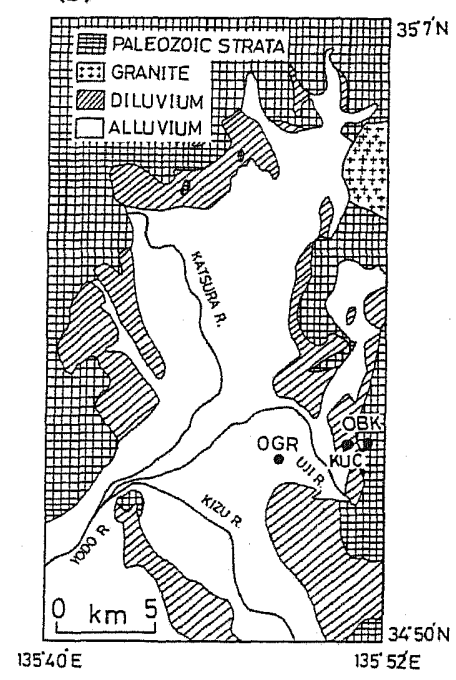

Fig. 1. (a) Map showing the locations of the Kyoto basin (shaded rectangular area) and the epicenter of the western Nagano Prefecture earthquake (solid circle). (b) Geological map of the Kyoto basin. The area is an enlargement of the shaded area in (a). Solid circles indicate locations of seismic observation sites. (c) Tripartite-array configuration at site OGR.

Table 1. Aftershock events of the western Nagano Prefecture earthquake analyzed in this study.

\begin{tabular}{|c|c|c|c|c|c|c|c|c|}
\hline No. & Year & M D & $\mathrm{h}: \min$ & $\mathrm{s}$ & Lat. & Long. & $\begin{array}{c}\text { Depth } \\
(\mathrm{km})\end{array}$ & Mag. \\
\hline 1 & 1984 & 914 & $10: 31$ & 9.3 & $35^{\circ} 49.3^{\prime}$ & $137^{\circ} 30.5^{\prime}$ & 12 & 3.8 \\
\hline 2 & 1984 & 914 & $12: 42$ & 28.2 & 3548.7 & 13731.4 & 11 & 3.8 \\
\hline 3 & 1984 & 914 & $13: 41$ & 7.4 & 3549.7 & 13735.1 & 8 & 4.0 \\
\hline 4 & 1984 & 915 & $8: 16$ & 20.0 & 3550.2 & 13736.1 & 9 & 4.2 \\
\hline 5 & 1984 & 915 & $16: 08$ & 32.8 & 3550.7 & 13734.1 & 8 & 4.1 \\
\hline
\end{tabular}

As can be seen from Fig. 1(b), the Kyoto basin is oriented almost north-south. Hence, its subsurface structure is assumed to be approximately two-dimensional in the EW cross section. Therefore, if an epicenter of an earthquake is located to the east or the west of the seismic observation area, then we can compute seismic responses in the context of the two-dimensional problem. The aftershock events of the 1984 western Nagano Prefecture earthquake satisfied the above condition best of all the events recorded by the array. Hence we will use five of these aftershock events, whose data quality is high, for the analysis and simulation in the following 


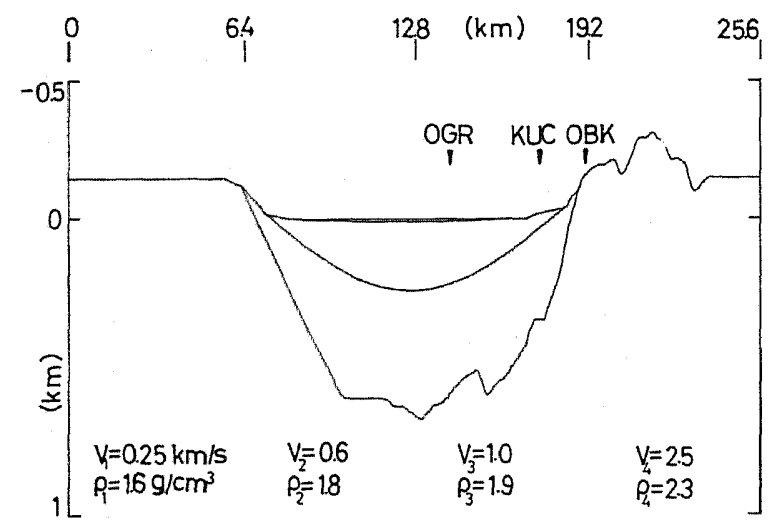

Fig. 2. E-W cross section of the subsurface structural model of the Kyoto basin. Solid triangles indicate the locations of the sites. $V_{i}$ and $\rho_{i}$ denote the $\mathrm{S}$ wave velocity and the density in the $i$-th layer.

sections; they are listed in Table 1. For the last three events, however, no records were obtained at subsite Sl of the tripartite subarray.

In this area, various geophysical surveys have been conducted since the beginning of the 1970's. We constructed a subsurface structural model of the Kyoto basin as shown in Fig. 2, referring to these exploration results. The S-wave velocity of the bedrock was adopted from AKamatsu et al. (1975). The shape of the basinbedrock interface was from KITSUNEZAKI et al. (1971). The S-wave velocities, densities, and shapes of interfaces within the basin were from HORIKE (1985) with slight modifications.

\section{Analysis of Seismic Array Data}

\subsection{Features of shear waves in the Kyoto basin}

Figures 3(a) and 4(a) show original NS-component seismograms of the events No. 2 and No. 3 in Table 1, respectively. Comparing the traces at the three sites, we notice the following three features: (1) In the first part of the $S$ wave (from the onset of the $S$ wave to a few seconds later), the seismic waves at the basin sites, $\mathrm{KUC}$ and OGR, are almost equal in amplitude and much larger than those at the rock site, OBK. (2) In the later part of the S wave, the seismic waves at KUC decay in amplitude rapidly, but those at OGR remain large for a long time. (3) The seismic waves at OGR are richer in high-frequency components (above $3 \mathrm{~Hz}$ ) than those at KUC.

Since high-frequency components greatly influence the seismogram at OGR, we wish to determine whether the first two features are also present at lower frequencies. Figures 3(b) and 4(b) show low-pass-filtered seismograms of events No. 2 and No. 3, respectively. We can see feature 2 more distinctly in the low-pass- 
(a)
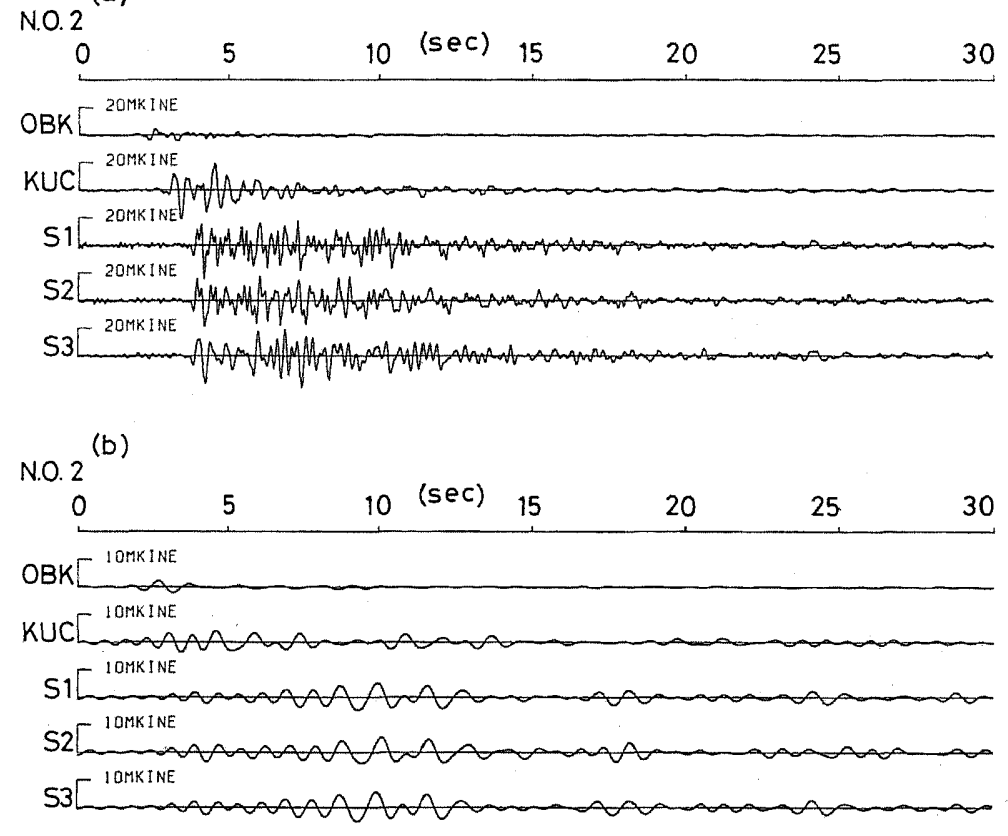

Fig. 3. (a) Original NS-component seismograms of event No. 2. They are displayed from a few seconds earlier than the $\mathrm{S}$ wave onset. (b) Low-passfiltered $(1.4 \mathrm{~Hz})$ seismograms of event No. 2.

filtered seismograms than in the original ones. However, feature 1 is not always true. So we modify it for the low-pass-filtered seismograms as follows. (1') In the first part of the $\mathrm{S}$ wave, the seismic waves at KUC are amplified more than those at OGR.

Since high-frequency components at the site OGR are supposed to be greatly amplified by the surface soft thin layer of the subsurface model in Fig. 2 and to be little influenced by the irregular basin-bedrock interface and, in addition, it is difficult to simulate such high-frequency components using the AL method, we will focus on features $1^{\prime}$ and 2 for the low-pass-filtered seismograms, i.e., the spatial waveform variation and elongation of duration of the S-wave portion, in the following study.

\subsection{Multiple-filter analysis of tripartite-subarray data}

In order to understand the cause of the spatial waveform variation and elongation of duration, we will examine what kinds of waves (surface waves or body waves) are contained in the S-wave portion. As is well known, the phase velocity of surface waves trapped within a basin is lower than the S-wave velocity of the bedrock, while the phase velocity of body waves (apparent velocity) is higher than that (EwING et al., 1957). The S-wave velocity of the bedrock below the Kyoto basin 

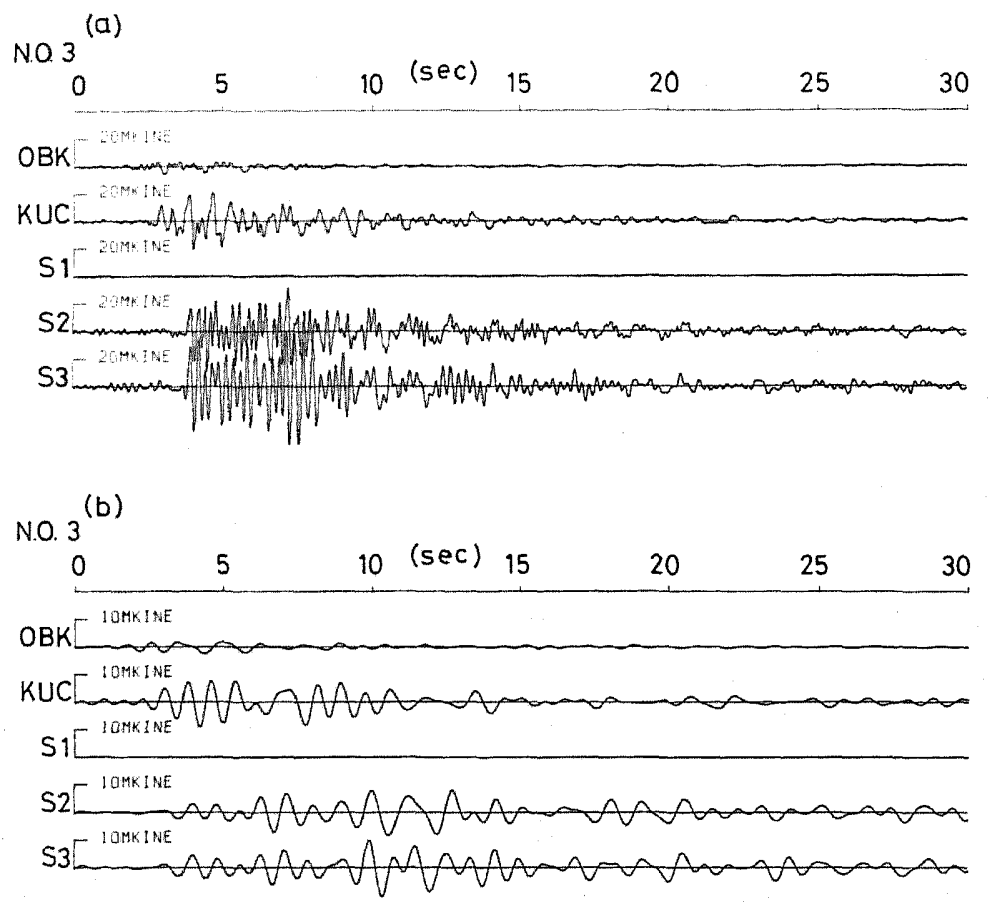

Fig. 4. (a) Original NS-component seismograms of event No. 3. (b) Low-passfiltered $(1.4 \mathrm{~Hz})$ seismograms of event No. 3 .

is $2.5 \mathrm{~km} / \mathrm{s}$, as shown in Fig. 2. Thus, we have a criterion for discriminating between surface waves and body waves, i.e., a wavelet whose phase velocity is lower than $2 \mathrm{~km} / \mathrm{s}$ is assumed to be a surface wave.

We estimated phase velocities of the wavelets contained in the S-wave portion of events No. 1 and No. 2. Figure 5 shows the results of the application of a Gaussian multiple-filter technique (DzIEwONSKI et al., 1969) to the seismograms of event No. 2. The wavelets denoted by the capital letters $A$ lie in the first part of the $S$ wave and those by $B$ in the later part. The phase velocities and propagation directions of these wavelets were computed from the time differences of corresponding peaks and troughs, and are shown by arrows in the circle. Phase velocities higher than $3 \mathrm{~km} / \mathrm{s}$ are displayed using a $3 \mathrm{~km} / \mathrm{s}$ arrow because of the lack of resolving power of the tripartite array for such high phase-velocities. At all frequencies except $1.11 \mathrm{~Hz}$, the wavelets denoted by A propagate at phase velocities higher than $3 \mathrm{~km} / \mathrm{s}$ from the approximate direction of the epicenter. Hence these wavelets are considered to be the direct $S$ wave from the earthquake source. The disagreement between propagation direction and epicenter direction at $1.11 \mathrm{~Hz}$ is probably due to the poor signal-to-noise ratio of wavelet $\mathrm{A}$. On the other hand, the phase velocities of the wavelets denoted by $B$ range from $0.9 \mathrm{~km} / \mathrm{s}$ to $1.9 \mathrm{~km} / \mathrm{s}$. Judging from the 
above-mentioned criterion, these wavelets in the later part are considered to be surface waves. However, since these phase velocities do not always decrease with frequency, these wavelets may belong to several modes of surface waves.

In summary, the phase velocities of the wavelets contained in the S-wave portion of event No. 2 suggest that the earlier part primarily contains direct $S$ waves and the later part contains surface waves. This result also applies for event No. 1. We further applied the multiple-filter technique to the last three events in Table 1. In these cases, we could not estimate the phase velocity because of the failure in recording at subsite $\mathrm{S} 1$. However, arrival-time differences between subsites $\mathrm{S} 2$ and S3 distributed in the same range as those of the first two events. That is, the wavelet in the first part arrives at two subsites S2 and S3 almost simultaneously, while the wavelets in the later part arrive at subsite S2 $0.05 \mathrm{~s}$ to $0.2 \mathrm{~s}$ later than at subsite S3. Therefore, the last three events support the results obtained from the first two events.

We will next examine where the surface waves in the later part of the S-wave portion are generated. We test the assumption that they are generated at the basinbedrock interface below the eastern margin of the basin when the direct $S$ waves are incident there. As can be seen from Fig. 2, the distance between site OGR and the eastern margin of the Kyoto basin is about $4 \mathrm{~km}$, and the direct $S$ waves are probably incident at the basin-bedrock interface at a time intermediate between the onsets of the $\mathrm{S}$ waves at sites OBK and KUC. We can thereby draw the group velocity axis shown below the seismograms at each frequency in Fig. 5. Using the axis, we can estimate the group velocities of prominent wavelets. As is well known, the group velocity of surface waves trapped in a basin is less than both the $S$ wave velocity of the bedrock and the corresponding phase velocity (EWING et al., 1957). We will compare the estimated group velocity with these two velocities. In Fig. 5(a), for example, at the frequency $0.62 \mathrm{~Hz}$, the group velocity of the wavelet B is about $0.5 \mathrm{~km} / \mathrm{s}$. This value is obviously lower than the corresponding phase velocity $(0.9 \mathrm{~km} / \mathrm{s})$ and the S-wave velocity of the bedrock $(2.5 \mathrm{~km} / \mathrm{s})$. At high frequencies in Fig. 5, the group velocities are also lower than these two velocities. This observation supports the assumption that the surface waves contained in the later part of the $S$ wave are generated mostly at the eastern margin of the Kyoto basin.

\section{Extension of the AL Method to Compute the Response of Subsurface Structure Having Multiple Irregular Interfaces}

\subsection{Computation method}

Using the multiple-filter technique, we have shown in the previous section that surface waves which were probably generated at the eastern margin of the Kyoto basin caused the spatial waveform variation and elongation of duration of the Swave portion. We will investigate whether the subsurface model of the Kyoto basin as shown in Fig. 2 can generate surface waves at the margin of the basin having the features discussed in section 3 and the correct amplitudes. This will be done using 
(a)

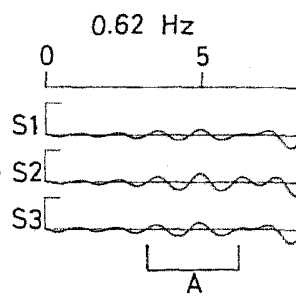

G. Vel.

08

0.4

$0.27 \mathrm{~km} / \mathrm{s}$

$\begin{array}{ccccc}1.11 \mathrm{~Hz} & 10 \text { (sec) } 15 & 20 \\ 0 & 5 & 1\end{array}$ G. Vel.

0.8

0.4

$027 \mathrm{~km} / \mathrm{s}$

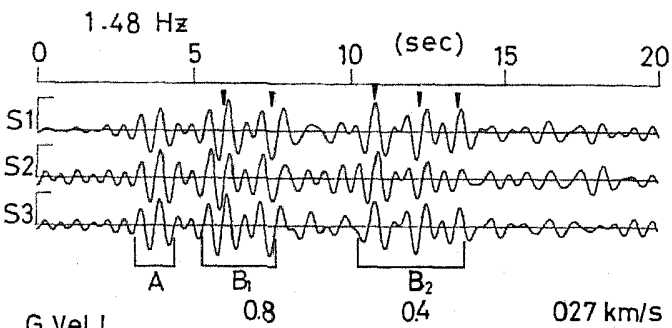

G Vel.

0.8

0.4

$027 \mathrm{~km} / \mathrm{s}$
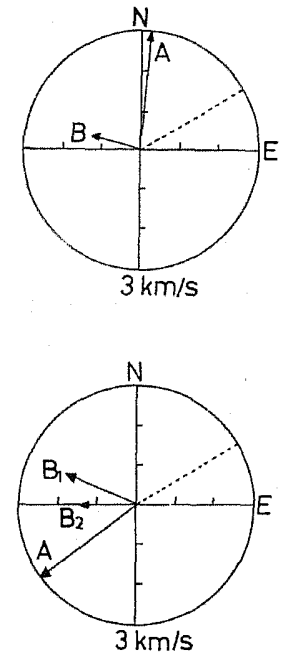

(d) 52[
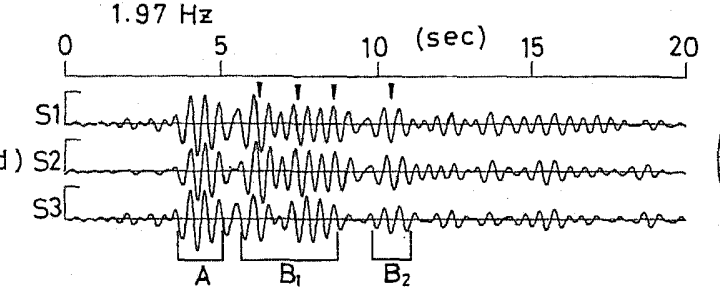
G.Vel. 0.4 $027 \mathrm{~km} / \mathrm{s}$

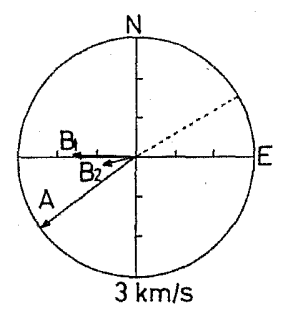

(e)

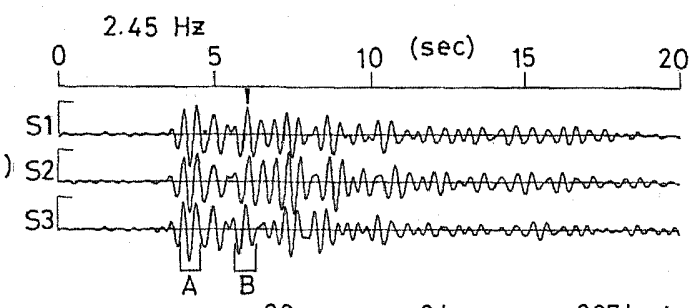

G. Vel. 0.8 0.4 $0.27 \mathrm{~km} / \mathrm{s}$

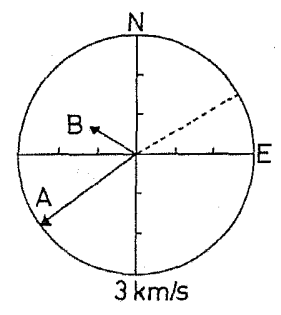

Fig. 5 
comparison between recorded and simulated seismograms. The simulation of seismic waves will be made by the AKI and LARNER (1970) method. However, while these authors gave the formulas only for structures with a single interface, there are three interfaces below the Kyoto basin. Hence we will extend the AL method to be applicable to structures with irregular interfaces following the suggestion in AKI and LARNER (1970) (see Appendix). KoHKETSU (1987) also devised the computation method of seismograms extending the reflectivity method to two-dimensionally layered media based on AKI and LARNER (1970).

As is well known, the AL method has several sources of numerical error (AKI and LARNER, 1970). In this study, we avoided large numerical errors by restricting the time response to a frequency band in which the frequency response changed smoothly.

\subsection{Numerical examples}

We will compute responses of the two subsurface structural models shown in Fig. 6 to an incident plane SH Ricker wavelet using the AL method described in Appendix. The incident $\mathrm{SH}$ motion $W(t)$ is expressed as

$$
W(t)=\frac{\sqrt{\pi}}{2}\left(\left|\frac{\pi\left(t-t_{0}\right)}{2}\right|^{2}-0.5\right) \exp \left(-\left|\frac{\pi\left(t-t_{0}\right)}{2}\right|^{2}\right) .
$$

Figure 7(a) shows the responses of the two-layer model to vertical incidence. Since the problem has been investigated in detail by BARD and BOUCHON (1980), we describe the features of computed seismograms briefly. We can see three prominent phases. Phase A is obviously an amplified SH body wave. Phase B is a surface wave, since the phase velocity is about $1.1 \mathrm{~km} / \mathrm{s}$, which is less than the S-wave velocity of the bedrock $(2.4 \mathrm{~km} / \mathrm{s})$, and the amplitude decays with depth. We can also see that it is generated at the left margin of the basin, since it begins to separate from phase $A$ at the left margin of the basin. For the same reason, phase $\mathrm{C}$ is a surface wave generated at the right margin of the basin. Figure 7(b) shows the responses of the three-layer model, where a surface layer with low velocity overlies the two-layer model, to vertical incidence. We again find the three prominent wavelets, consisting of $\mathrm{SH}$ body waves and surface waves generated at both margins of the basin. In both figures, we notice that surface waves generated at margins cause the spatial

Fig. 5. Estimation of phase and group velocities of wavelets contained in the Swave portion from the tripartite-array data of event No. 2 by the Gaussian multiple-filter technique. (a) At the center frequency $0.62 \mathrm{~Hz}$. Arrows in the circle show the phase velocity and propagation direction of a prominent wavelet denoted by the capital letters. Broken line indicates the direction of the epicenter of the western Nagano Prefecture earthquake. The axis G. vel. is for the measurement of the group velocity. Solid triangle indicates the group arrival time of the wavelet. (b) At the center frequency $1.11 \mathrm{~Hz}$. (c) At the center frequency $1.48 \mathrm{~Hz}$. (d) At the center frequency $1.97 \mathrm{~Hz}$. (e) At the center frequency $2.45 \mathrm{~Hz}$. 

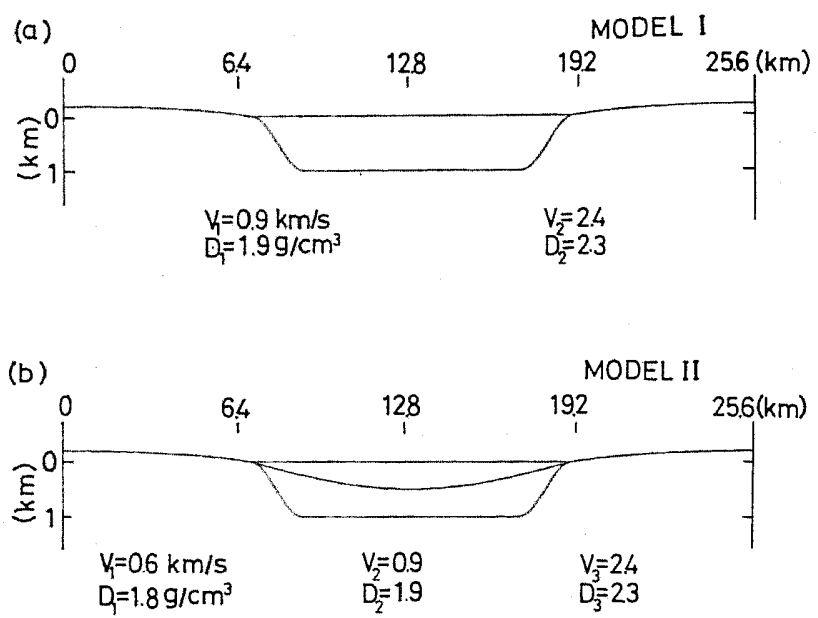

Fig. 6. Two subsurface structural models of sedimentary basins for simulation. $V_{i}$ and $D_{i}$ denote the shear velocity and density in the $i$-th layer. (a) Two-layer model. (b) Three-layer model.

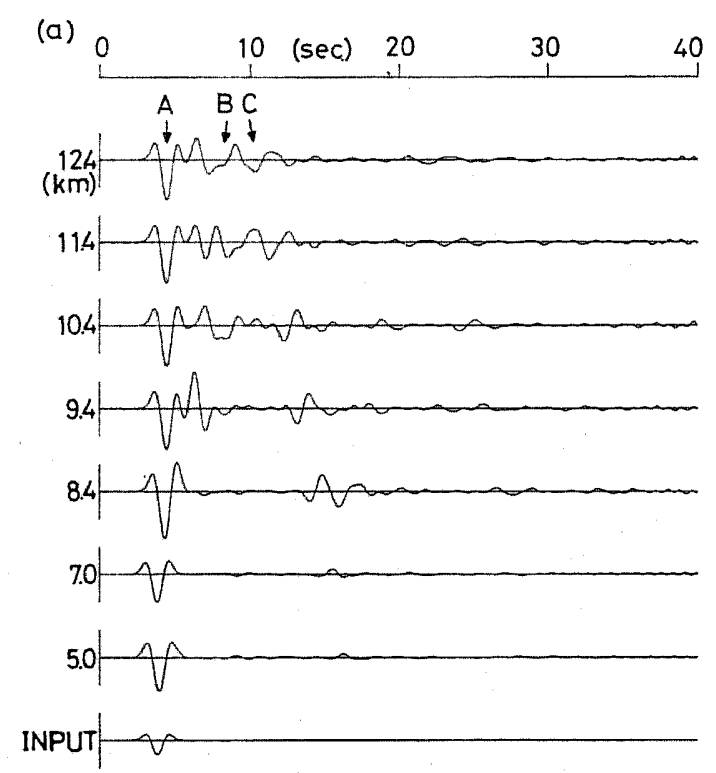

Fig. 7

waveform variation and elongation of duration and, in addition, that SH-bodywave amplitudes at the margin of the basin $(8.4 \mathrm{~km})$ are larger than those at the center $(12.8 \mathrm{~km})$. The latter observation is consistent with feature $1^{\prime}$ described in section 3 and is explained as follows. At the site near the basin margin, the surface waves, which begin to be excited there, superpose on the amplified SH body waves, 

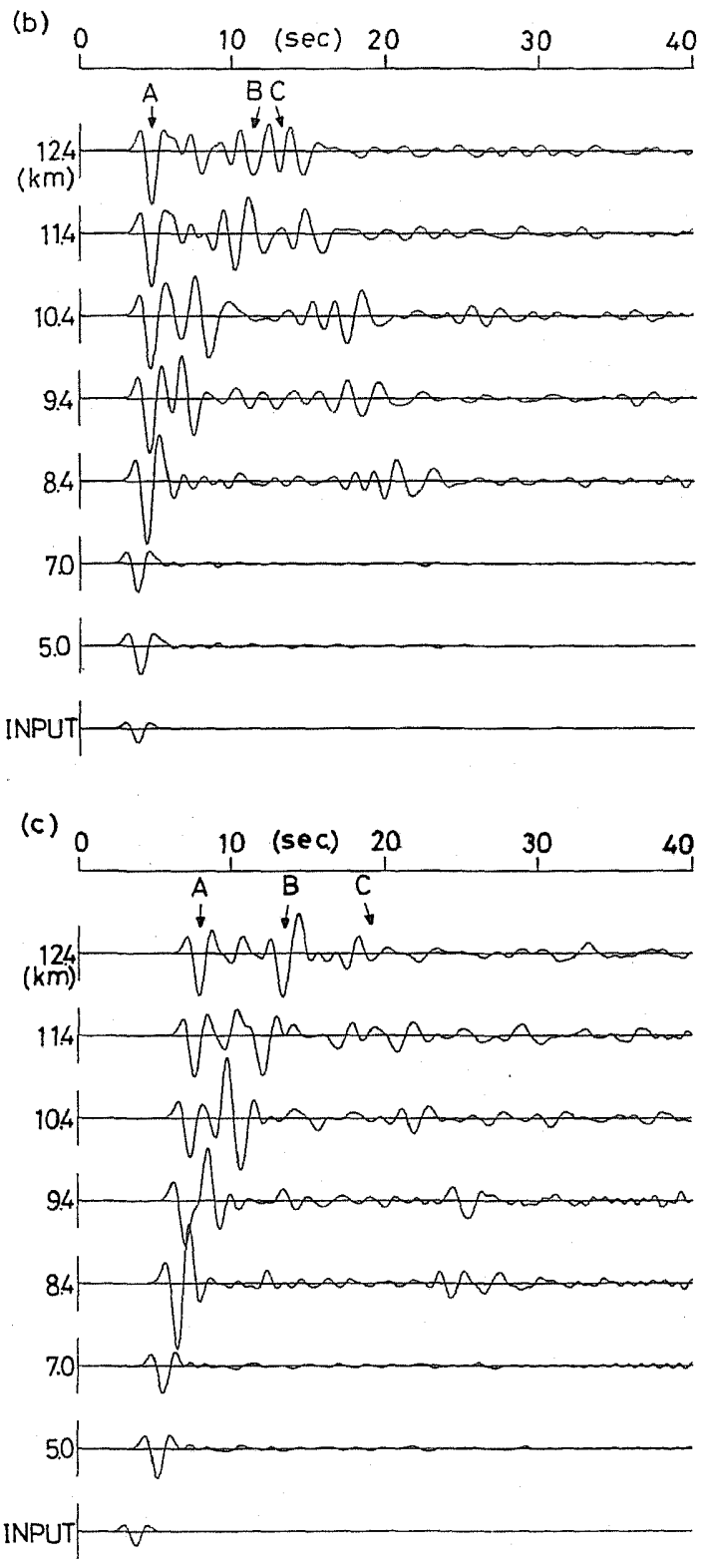

Fig. 7. (a) Responses of the two-layer model in Fig. 6(a) to a vertically incident $\mathrm{SH}$ wave. Numbers in front of seismic traces denote horizontal distances on the free surface. (b) Responses of the three-layer model to a vertically incident SH wave. (c) Responses of the three-layer model shown in Fig. 6(b) to an obliquely incident $\left(40^{\circ}\right) \mathrm{SH}$ wave. 
while at the center of the basin the surface waves separate from the SH body waves. The difference between the responses of the two structural models is that in the three-layer model the body waves are amplified more and the surface waves are excited more than those in the two-layer model. Consequently, the spatial waveform variation and elongation of duration in the three-layer model is much greater than in the two-layer model. We should note that in the recorded seismograms, surface waves were generated mostly at the eastern margin, while in the two numerical examples they are generated at both margins. This discrepancy may be explained by the effect of incident angle to some extent. Figure 7 (c) shows the responses to oblique incidence $\left(40^{\circ}\right)$. Phase B in Fig. 7(c) is larger than that in Fig. 7(b) and phase $\mathrm{C}$ in Fig. 7(c) is smaller than that in Fig. 7(b). This fact means that larger surface waves are generated at the side of the incidence than at the other side. The above three examples imply that oblique incidence of $S$ waves from the east may explain the recorded seismograms fairy well.

\section{Comparison between Recorded and Synthetic Seismograms}

So far, we have showed from the multiple-filter analysis and the numerical simulations that in a basin-like structure surface waves generated at the margin cause seismic waveform variation and elongation of duration of the S-wave portion. In this section, we will study whether synthetic seismograms computed by the AL method can reproduce the amplitudes and the above-mentioned features of the recorded seismograms.

Previously, the prediction of seismic motions has often been made using the HASKELL method (1960) in which a subsurface structure is assumed to be flat layered (for example, NARUSE et al., 1984; OMOTE and WATANABE, 1984). Therefore, it is of interest to engineering seismology to compare synthetic seismograms calculated using the Haskell method with recorded ones. Figure 8(a) and (b) shows synthetic seismograms calculated using the Haskell method corresponding to events No. 2 and No. 3, respectively. The incident angle was $-30^{\circ}$. This value was estimated by the AL method and explained the time difference between the onsets of the $\mathrm{S}$ wave at the two sites OBK and KUC (about $0.5 \mathrm{~s}$, see Figs. 3(a) and 4(a)). In this computation, the flat layered subsurface model at each site is adopted from the vertical profile just below the site in the model shown in Fig. 2. The incident plane SH motions were estimated in such a way that the recorded seismogram on the rock outcrop (OBK) agreed well. As can be seen from Fig. 8, synthetic seismograms in the basin scarcely exhibit the spatial waveform variation and elongation of duration because of non-excitation of surface waves, and obviously do not reproduce the recorded seismograms in Figs. 3(b) and 4(b). This fact means that the Haskell method (or flat layered model) is not suitable for the prediction of seismic motions in a basin-like structure.

Figure 9(a) and (b) shows synthetic seismograms corresponding to events No. 2 and No. 3, respectively, calculated using the AL method. The incident plane $\mathrm{SH}$ 
(a)

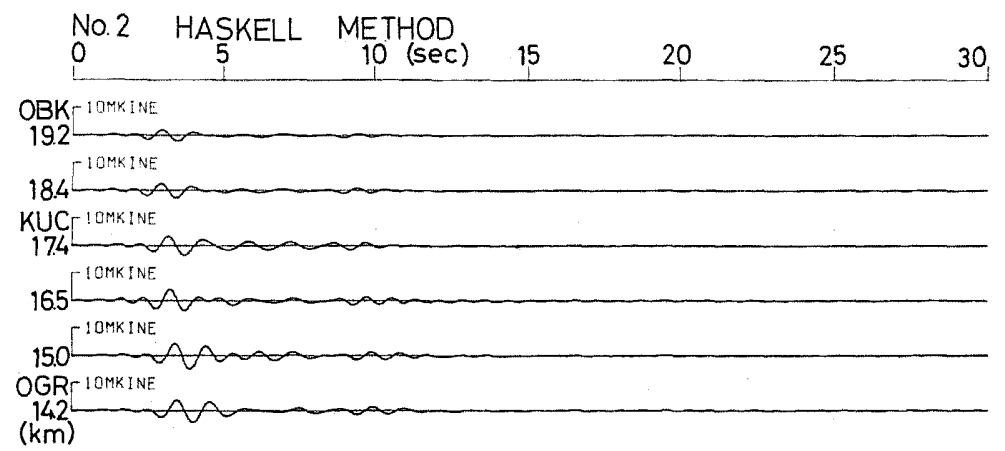

(b)

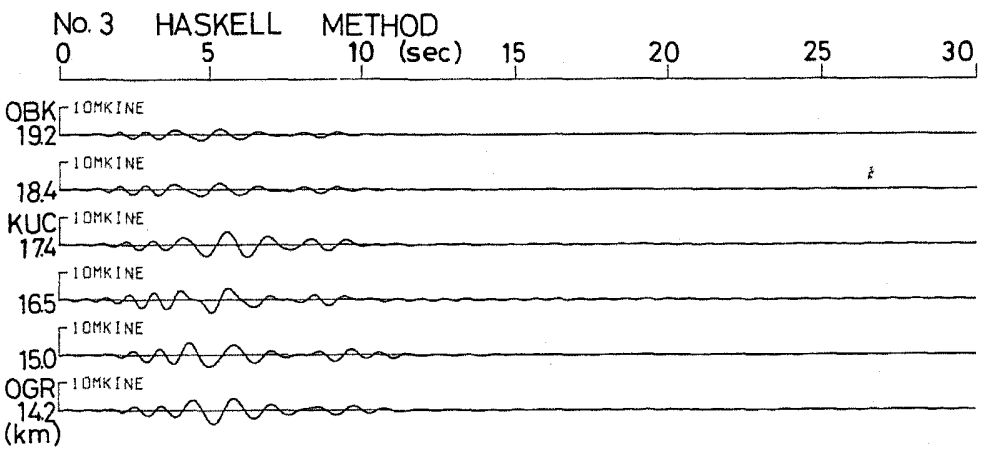

Fig. 8. (a) Synthetic seismograms of the Kyoto basin calculated using the Haskell method. The input motions were inferred from the recorded seismogram at site OBK of event No. 2. (b) Same figure as Fig. 8(a) except that the input motions were inferred from event No. 3 .

motions were estimated by the same procedure as in the Haskell method. The synthetic seismograms in the basin exhibit the spatial waveform variation and elongation of duration due to surface-wave generation. This fact again means that the prediction of seismic motions in a basin-like structure requires the treatment of irregular interfaces. However, compared with the recorded seismograms, the excitation of surface waves is not sufficient and consequently the duration is shorter than that of the recorded seismograms. This fact probably means that we have to include the effects of three-dimensional structure and non-plane wave front of incident waves to explain the recorded seismograms in amplitudes more completely.

\section{Conclusion}

We obtained the following conclusions from the analyses of seismograms recorded by the array in the Kyoto basin and the seismic wave simulations. 
(a)

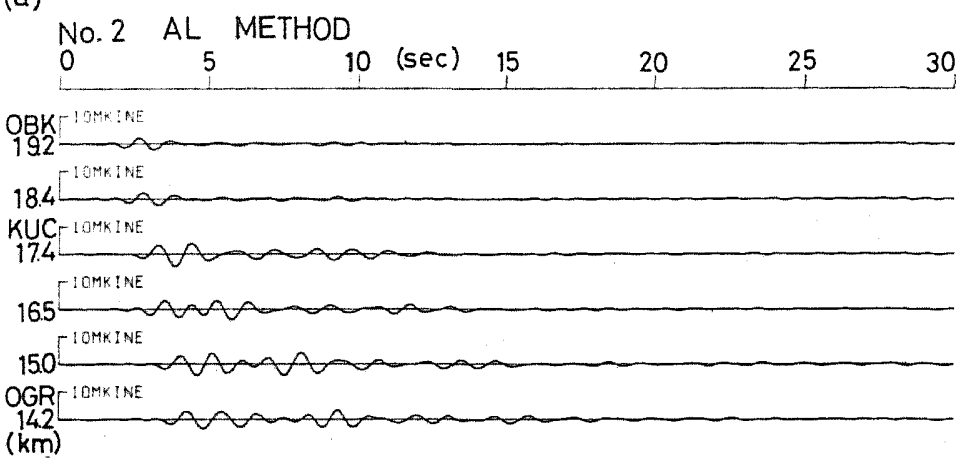

(b)

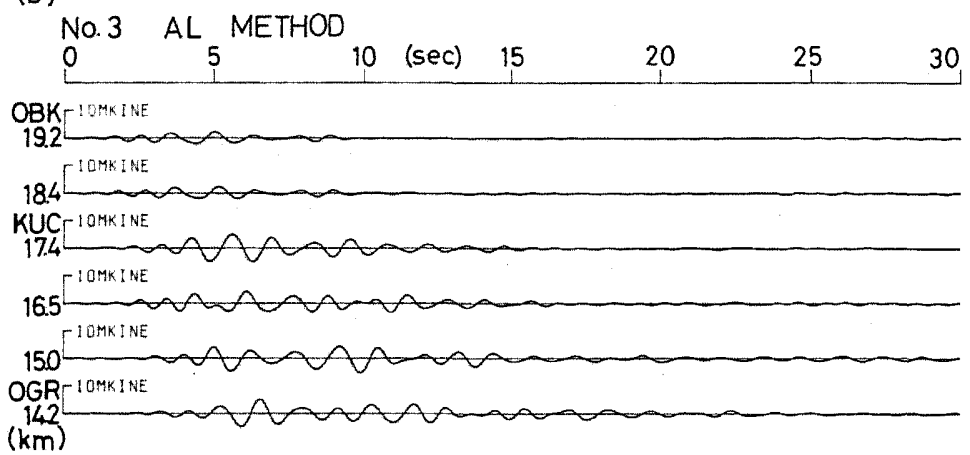

Fig. 9. (a) Synthetic seismograms of the Kyoto basin calculated using the AL method. The input motions were inferred from the recorded seismogram at site OBK of event No. 2. (b) Same figure as Fig. 9(a) except that the input motions were inferred from event No. 3 .

(1) The seismic motions observed in the Kyoto basin exhibit the feature that the S-wave portion spatially changes in waveform and elongates in duration due to surface waves which are probably excited at the eastern margin of the basin.

(2) Synthetic seismograms for a two-dimensional structure with irregular interfaces exhibit this feature, but are smaller in amplitudes compared with recorded seismograms, primarily because of less excitation of surface waves.

(3) Synthetic seismograms calculated using the Haskell method scarcely show the observed features and are unsatisfactory in explaining the recorded seismograms.

I thank Y. Kobayashi and Paul G. Somerville for critical reading of the manuscript and invaluable suggestions for improving it, and $\mathrm{K}$. Irikura and Y. Shinozaki for useful discussions about the numerical computations. I also thank P.-Y. Bard for valuable 
suggestions for improving the manuscript and T. Iwata, T. Idei, and M. Hoshiba for their aid in seismic observations. All computations were run on the FACOM VP-200 at the Data Processing Center of Kyoto University.

\section{REFERENCES}

Akamatsu, J., T. Furuzawa, and K. Irikura, On the nature of $\mathrm{S}$ waves from local small earthquakes observed at Amagase Crustal Movement Observatory, Annu. Disas. Prev. Res. Inst., Kyoto Univ.,18-B, 11-21, 1975 (in Japanese).

AKI, K. and K. L. LARNER, Surface motion of a layered medium having an irregular interface due to incident plane SH waves, J. Geophys. Res., 75, 933-954, 1970.

BARD, P. -Y. and M. BouchoN, The seismic response of sediment-filled valleys. Part 1. The case of incident SH waves, Bull. Seismol. Soc. Am., 70, 1263-1286, 1980.

BARD, P.-Y. and M. BouCHON, The two-dimensional response of sediment-filled valleys, Bull. Seismol. Soc. Am., 75, 519-541, 1985.

BOORE, D. M., Finite difference methods for seismic wave propagation in heterogeneous materials, Methods Comput. Phys., 12, 1-36, 1972.

Červený, V., J. Pleinerová, L. Klimeś, and I. Pšenčík, High-frequency radiation from earthquake source in laterally varying structures, Geophys. J. R. Astron. Soc., 88, 43-79, 1987.

DRAVINSKY, M., Scattering of plane harmonic SH wave by dipping layer of arbitrary shape, Bull. Seismol. Soc. Am., 73, 1303-1319, 1983.

Dziewonski, A., S. Bloch, and M. Landisman, A technique for the analysis of transient seismic signals, Bull. Seismol. Soc. Am., 59, 427-444, 1969.

EWING, M., W. JARDETZKY, and F. PRess, Elastic Waves in Layered Media, McGraw-Hill, New York, 1957.

Haskell, N. A., Crustal reflection of plane SH waves, J. Geophys. Res., 65, 4147-4150, 1960.

HORIKE, M., Inversion of phase velocity of long-period microtremors to the S-wave-velocity structure down to the basement in urbanized areas, J. Phys. Earth, 33, 59-96, 1985.

KING, J. L. and B. E. TUCKER, Observed variation of earthquake motion over sediment-filled valleys, Bull. Seismol. Soc. Am., 72, 987-998, 1984.

KInoshita, S., The earthquake response characteristics of a thick sedimentary layer estimated by means of deep borehole observation, Rep. Nat. Res. Cent. Disas. Prev., 38, 25-154, 1985 (in Japanese).

KITSUNEZAKI, C., N. Goto, and Y. IWASAKI, Underground structure of the southern part of the Kyoto basin obtained from seismic explorations and some related problems of earthquake engineering, Annu. Disas. Prev: Res. Inst., Kyoto Univ., 14-A, 203-215, 1971 (in Japanese).

KoHKETSU, K., 2-D reflectivity method and synthetic seismograms for irregularly layered structure-1. SH-wave generation, Geophys. J. R. Astron. Soc., 89, 821-838, 1987.

KUDO, K., The study on the contribution of surface waves to strong ground motions, Proc. 7th WCEE, 499-506, 1980.

LiU, H.-L. and T. Heaton, Array analysis of the ground velocities and accelerations from the 1971 San Fernando, California, earthquake, Bull. Seismol. Soc. Am., 74, 1951-1968, 1984.

LYSMER, J. and L. A. DRAKE, The propagation of Love waves across nonhorizontally layered 
structures, Bull. Seismol. Soc. Am., 61, 1233-1252, 1971.

Minamishima, M., K. YAMAZAKI, and K. KuDo, A preliminary analysis of wave groups found in the strong ground motion records observed on sedimentary layers, Zisin (J. Seismol. Soc. Jpn.), Ser. 2, 39, 533-554, 1986 (in Japanese).

Naruse, S., A. Yoshida, K. Masaki, H. Kagami, M. Miyazaki, and A. Kubodera, Observation of 1 - to 5 -sec microtremors and their application to earthquake engineering. Part IX. An extended observation in the Nohbi plain, central Japan, Zisin (J. Seismol. Soc. Jpn.), Ser 2, 37, 383-395, 1984 (in Japanese).

OMOTE, S. and M. WATANABE, A note on the relation between the thickness of the soft surficial deposits and the maximum amplification at the surface, Butsuritanko, 37, 86-97, 1984 (in Japanese).

SANChez-Sesma, F. J. and J. A. Esquivel, Ground motion on alluvial valleys under incident plane SH waves, Bull. Seismol. Soc. Am., 69, 1107-1120, 1979.

Sasatani, T., A study of the strong motion of the western Hidaka earthquake, Geophys. Bull. Hokkaido Univ, 46, 69-83, 1985 (in Japanese).

TORIUMI, I., Characteristics of seismic motions in the Osaka plain, Proceedings of the Kinki Branch of the Architectural Society of Japan, 487-488, 1980 (in Japanese).

TRIFUnAC, M. D., Surface motion of a semi-cylindrical alluvial valley for incident plane SH waves, Bull. Seismol. Soc. Am., 61, 1755-1770, 1971.

\section{APPENDIX}

In this section we extend the Aki and Larner method to allow the computation of seismic responses of a two-dimensional basin-like structure having irregular interfaces and irregular topography. We consider the subsurface structure shown in Fig. A1. To the extent possible, the notations are the same as those in AKI and LARNER (1970). The displacement in the $i$-th layer is expressed as

$$
U_{i}=\int_{-\infty}^{\infty}\left(A_{i}(k) \mathrm{e}^{j v_{i} z}+B_{i}(k) \mathrm{e}^{-j v_{i} z}\right) \mathrm{e}^{j k x} \mathrm{~d} k
$$

and that in the half space as

$$
U=\mathrm{e}^{j\left(k_{0} x-v_{0} z\right)}+\int_{-\infty}^{\infty} A_{m}(k) \mathrm{e}^{j\left(k x+v_{m} z\right)} \mathrm{d} k
$$

where $j=\sqrt{-1}$, and $k$ and $v_{i}$ are horizontal and vertical wavenumbers in the $i$-th layer, respectively, and $k_{0}$ and $v_{0}$ refer to the incident wave.

We will first derive simultaneous linear equations of the coefficients $A$ and $B$ in the first layer. Substituting Eq. (1) into the free surface condition, we obtain a relation between these coefficients. Assuming a spatial periodicity $L$ of interfaces in the horizontal direction, then the above relation is satisfied not for continuous wavenumber but for discrete wavenumber $k=2 \pi n / L$ ( $n$ : integer) (AKI and LARNER, 1970) and is expressed in an infinite-sum equation. Approximating it by a $(2 N+1)$ finitesum equation, we obtain 


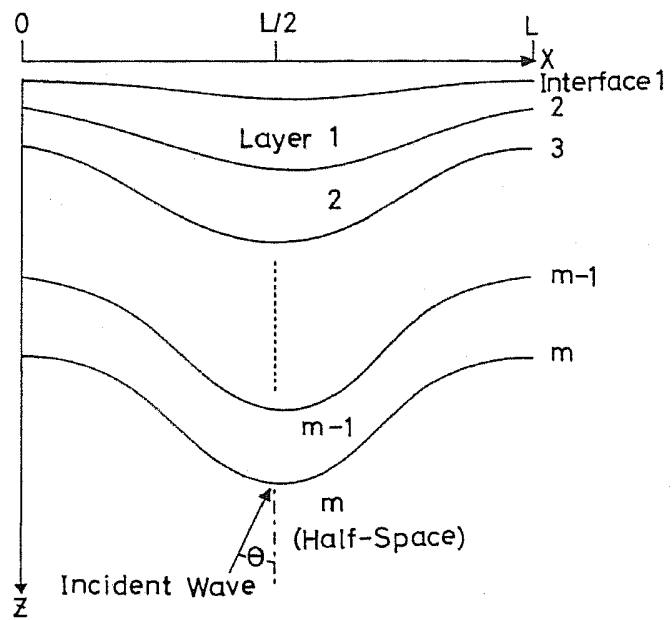

Fig. A1. Schematic subsurface structure with multiple irregular interfaces. The incident angle of the input motion is measured clockwise from the positive $Z$ direction.

$$
\sum_{n=-N}^{N}\left(A_{n}^{(1)} g_{n}^{(1,3)}(x)+B_{n}^{(1)} g_{n}^{(1,4)}(x)\right) \mathrm{e}^{j k_{n} x}=h^{(1)}(x)
$$

where $A_{n}^{(1)}=A_{1}\left(k_{n}\right) \Delta k, B_{n}^{(1)}=B_{1}\left(k_{n}\right) \Delta k, \Delta k=2 \pi / L$. The functions $g_{n}^{(1,3)}(x), g_{n}^{(1,4)}(x)$, and $h^{(1)}(x)$ are

$$
\begin{aligned}
& g_{n}^{(1,3)}(x)=\left(k_{n} N_{x}^{(1)}+v_{n}^{(1)} N_{z}^{(1)}\right) \mu^{(1)} \mathrm{e}^{j v_{n}^{(1)} \zeta^{(1)}} \\
& g_{n}^{(1,4)}(x)=\left(k_{n} N_{x}^{(1)}-v_{n}^{(1)} N_{z}^{(1)}\right) \mu^{(1)} \mathrm{e}^{-j v_{n}^{(1)} \zeta^{(1)}} \\
& h^{(1)}(x)=0
\end{aligned}
$$

respectively. The vector $\left(N_{x}^{(i)}, N_{z}^{(i)}\right)$ and $\zeta^{(i)}$ denote the unit normal vector and the depth of the $i$-th interface, respectively. $\mu^{(i)}$ is the rigidity in the $i$-th layer. Equation (3) is spatially Fourier transformed and then simultaneous linear equations are obtained. They are written in simplified form as

$$
G^{(1)} A^{(1)}=H^{(1)}
$$

where the vector $\boldsymbol{H}^{(1)}$ is the $(2 N+1)$ dimensional zero vector and the vector $A^{(i)}$ is defined as

$$
\left(A^{(i)}\right)^{T}=\left(A_{-N}^{(i)}, \cdots, A_{0}^{(i)}, \cdots, A_{N}^{(i)}, B_{-N}^{(i)}, \cdots, B_{0}^{(i)}, \cdots, B_{N}^{(i)}\right) .
$$

The matrix $G^{(1)}$ is expressed, using $(2 N+1)(2 N+1)$ dimensional submatrices $G^{(1.3)}$ and $G^{(1,4)}$, as

$$
G^{(1)}=\left(G^{(1,3)} G^{(1,4)}\right)
$$

where an element of the submatrix $G^{(i, t)}$ is defined as 


$$
G_{n, k}^{(i, l)}=\frac{1}{L} \int_{0}^{L} g_{n}^{(i, l)}(x) \mathrm{e}^{j 2 \pi(n-k) x / L} \mathrm{~d} x .
$$

We will derive relations between coefficients in the $i$-th layer and those in the $(i-1)$-th layer. From the conditions of the continuity of displacement and stress at the $i$-th interface, we obtain

$$
\begin{aligned}
& \sum_{n=-N}^{N}\left(A_{n}^{(i-1)} g_{n}^{(i-1,1)}(x)+B_{n}^{(i-1)} g_{n}^{(i-1,2)}(x)\right) \mathrm{e}^{j k_{n} x}=\sum_{n=-N}^{N}\left(A_{n}^{(i)} g_{n}^{(i, 1)}(x)+B_{n}^{(i)} g_{n}^{(i, 2)}(x)\right) \mathrm{e}^{j k_{n} x} \\
& \sum_{n=-N}^{N}\left(A_{n}^{(i-1)} g_{n}^{(i-1.3)}(x)+B_{n}^{(i-1)} g_{n}^{(i-1,4)}(x)\right) \mathrm{e}^{j k_{n} x}=\sum_{n=-N}^{N}\left(A_{n}^{(i)} g_{n}^{(i, 3)}(x)+B_{n}^{(i)} g_{n}^{(i, 4)}(x)\right) \mathrm{e}^{j k_{n} x}
\end{aligned}
$$

where

$$
\begin{aligned}
& g_{n}^{(i .1)}(x)=\mathrm{e}^{j v_{n}^{(i)} \zeta^{(i)}} \\
& g_{n}^{(i .2)}(x)=\mathrm{e}^{-j v_{n}^{(i) \xi^{(i)}}} \\
& g_{n}^{(i .3)}(x)=\mu^{(i)}\left(k_{n} N_{x}^{(i)}+v_{n}^{(i)} N_{z}^{(i)}\right) \mathrm{e}^{j v_{n}^{(i) \zeta^{(i)}}} \\
& g_{n}^{(i .4)}(x)=\mu^{(i)}\left(k_{n} N_{x}^{(i)}-v_{n}^{(i)} N_{z}^{(i)}\right) \mathrm{e}^{-j v_{n}^{(i)} \zeta^{(i)}}
\end{aligned}
$$

Applying the same procedure to Eqs. (5) and (6) as was used to obtain Eq. (4) from Eq. (3), we obtain

$$
G_{s}^{(i)} A^{(i)}=G_{d}^{(i-1)} A^{(i-1)}
$$

where the matrix $G_{d}^{(i)}$ is defined at the lower (deeper) interface of the $i$-th layer and the matrix $G_{s}^{(i)}$ at the upper (shallower) interface, respectively. The matrix $G^{(i)}$ is expressed, using $(2 N+1)(2 N+1)$ dimensional submatrices $G^{(i, 1)}, G^{(i, 2)}, G^{(i, 3)}, G^{(i, 4)}$, as

$$
\cdot G^{(i)}=\left(\begin{array}{c:c}
G^{(i, 1)} & G^{(i, 2)} \\
\hdashline G^{(i .3)} & G^{(i .4)}
\end{array}\right) \quad(i=2,3, \cdots, m-1) .
$$

Changing the form of Eq. (7) into the form $A^{(i)}=R^{(i)} A^{(i-1)}$, where $R^{(i)}=\left(G_{s}^{(i)}\right)^{-1} G_{d}^{(i-1)}$, and using it iteratively, we obtain

$$
A^{(m-1)}=R^{(m-1)} R^{(m-2)} \cdots R^{(2)} A^{(1)}=E A^{(1)} .
$$

This equation directly connects the coefficients in the first layer with those in the $(m-1)$-th layer.

We will next derive relations between coefficients in the $(m-1)$-th layer and those in the half-space. Using the conditions of the continuity of displacement and stress at the $(m-1)$-th interface, we get 


$$
\begin{aligned}
& \sum_{n=-N}^{N}\left(A_{n}^{(m-1)} g_{n}^{(m-1,1)}(x)+B_{n}^{(m-1)} g_{n}^{(m-1,2)}(x)\right) \mathrm{e}^{j k_{n} x}=h^{(2)}(x)+\sum_{n=-N}^{N} A_{n}^{(m)} g_{n}^{(m, 1)}(x) \mathrm{e}^{j k_{n} x} \\
& \sum_{n=-N}^{N}\left(A_{n}^{(m-1)} g_{n}^{(m-1,3)}(x)+B_{n}^{(m-1)} g_{n}^{(m-1,4)}(x)\right) \mathrm{e}^{j k_{n} x}=h^{(3)}(x)+\sum_{n=-N}^{N} A_{n}^{(m)} g_{n}^{(m, 3)}(x) \mathrm{e}^{j k_{n} x}
\end{aligned}
$$

where

$$
\begin{aligned}
& h^{(2)}(x)=\mathrm{e}^{-j v_{0} \zeta(m)} \\
& h^{(3)}(x)=\mu^{(m)}\left(N_{x}^{(m)} k_{0}-N_{z}^{(m)} v_{0}\right) \mathrm{e}^{-j v_{0} \zeta^{(m)}} .
\end{aligned}
$$

Again applying the same procedure to Eqs. (9) and (10) as was used to obtain Eq. (4) from Eq. (3), we obtain

$$
G_{d}^{(m-1)} A^{(m-1)}=H^{(m)}+G^{(m)} A^{(m)}
$$

where the vector $\boldsymbol{H}^{(m)}$ is defined as

$$
\left(\boldsymbol{H}^{(m)}\right)^{T}=\left(H_{-N}^{(2)}, \cdots, H_{0}^{(2)}, \cdots, H_{N}^{(2)}, H_{-N}^{(3)}, \cdots, H_{0}^{(3)}, \cdots, H_{N}^{(3)}\right) .
$$

The vectors $\boldsymbol{H}^{(2)}$ and $\boldsymbol{H}^{(3)}$ are Fourier transforms of $h^{(2)}$ and $h^{(3)}$, respectively. The vector $\boldsymbol{A}^{(m)}$ is defined as

$$
\left(A^{(m)}\right)^{T}=\left(A_{-N}^{(m)}, \cdots, A_{0}^{(m)}, \cdots, A_{N}^{(m)}\right)
$$

and the matrix $G^{(m)}$ is expressed, using submatrices $G^{(m .1)}, G^{(m .3)}$ as

$$
G^{(m)}=\left(\frac{G^{(m .1)}}{G^{(m .3)}}\right)
$$

We will finally derive simultaneous linear equations for the coefficients in the first layer and the half-space. Substituting Eq. (8) into Eq. (11), we obtain

$$
G_{d}^{(m-1)} E A^{(1)}-G^{(m)} A^{(m)}=H^{(m)} .
$$

Further, combining and arranging the Eqs. (4) and (12), we obtain simultaneous linear equations

$$
D A=H
$$

The matrix $D$ is defined as

$$
D=\left(\begin{array}{c:c}
G^{(1)} & 0 \\
\hdashline G_{d}^{(m-1)} E & -G^{(m)}
\end{array}\right)
$$

and the vectors $\boldsymbol{A}$ and $\boldsymbol{H}$ are given, respectively, as

$$
\begin{aligned}
& A^{T}=\left(\left(A^{(1)}\right)^{T},\left(A^{(m)}\right)^{T}\right) \\
& H^{T}=\left(\left(H^{(1)}\right)^{T},\left(H^{(m)}\right)^{T}\right)
\end{aligned}
$$


Putting the coefficients $A_{n}^{(1)}$ and $B_{n}^{(1)}$, solutions of the simultaneous Eq. (13), into the following equation

$$
U(\omega, x)=\sum_{n=-N}^{N}\left(A_{n}^{(1)} \mathrm{e}^{j v_{n}^{(1) \zeta(1)}}+B_{n}^{(1)} \mathrm{e}^{\left.-j v_{n}^{(1)}\right)(1)}\right) \mathrm{e}^{j k_{n} x}
$$

we obtain the frequency response $U(\omega, x)$ at distance $x$ on the free surface and angular frequency $\omega$. We can obtain the time responses by the inverse Fourier transform. 